\title{
Contradictory Concepts in Tortuosity Determination in Porous Media in Electrochemical Devices
}

\author{
Bernhard Tjaden ${ }^{1}$, Donal P. Finegan ${ }^{1}$, Jonathan Lane ${ }^{2}$, Dan J.L. Brett ${ }^{1}$, Paul R. Shearing ${ }^{1 *}$ \\ ${ }^{1}$ Electrochemical Innovation Lab, Department of Chemical Engineering, \\ University College London, WC1E 7JE, UK \\ ${ }^{2}$ Praxair, Inc., 175 E. Park Dr., Tonawanda, NY 14150, USA \\ *Corresponding author: e-mail: p.shearing@ucl.ac.uk
}

\begin{abstract}
Porous media are a vital component in almost every electrochemical device in the form of electrode, support or gas diffusion layers. Microstructural parameters of porous layers such as tortuosity, porosity and pore size diameter are of high importance and crucial for diffusive mass transport calculations. Among these parameters, the tortuosity remains ill-defined in the field of electrochemistry, resulting in a wide range of different calculation approaches. Here, we present a systematic approach of calculating the tortuosity of different porous samples using image and diffusion cell experimental-based methods. Image-based analyses include a selection of geometric and flux-based tortuosity calculation algorithms. Differences between the image and diffusion cell-based results are encountered and attributed to the small pore diameters and thereby induced Knudsen effects within the samples which govern the diffusion flux.
\end{abstract}

\section{Highlights}

- Microstructural analysis of oxygen transport membrane porous supports.

- Correlative tortuosity determinations via X-ray tomography and diffusion cell experiments.

- Evaluation of the effect of tortuosity, porosity and sample thickness on diffusion resistance.

- Visible differences between different tortuosity calculation approaches are encountered.

- Diffusion cell experiments yield the highest and geometric-based image quantification algorithms result in the lowest tortuosity values.

\section{Keywords}

- Tortuosity

- Simulation

- X-ray Tomography

- Diffusion cell experiments 


\section{Introduction}

The rate limiting step at high fuel utilisation ratios of oxygen transport membranes (OTMs) and fuel cells is dominated by mass transport limitations through porous layers ( $c f$. concentration losses in fuel cells) (Virkar et al., 2000; Wilson and Barnett, 2008; Shearing et al., 2013a). Here, microstructural characteristics of porous structures including tortuosity, porosity and pore size diameter, play a vital role in quantifying gaseous mass transport (Kast and Hohenthanner, 2000; Bertei et al., 2013; Yuan and Sundén, 2014).

At the same time, the mechanical stability of electrochemical devices during operation is ensured by porous support layers, commonly placed on the anode side. Such porous support layers can be several orders of magnitude thicker compared to the functional electrode and electrolyte layers (Tsai and Schmidt, 2011). Their mechanical strength is adjusted by altering either the microstructural properties, such as the porosity (Chen et al., 2015; Kaiser et al., 2016), or the thickness of the support layer. However, modifying these parameters can influence the mass transport behaviour and hence, the performance of the device.

The aforementioned microstructural characteristics are interrelated in a complicated manner (Robertson et al., 2010; Shearing et al., 2010), where tortuosity remains notoriously difficult to calculate (Tjaden et al.). In electrochemistry, the tortuosity is commonly used to quantify the resistance of a structure towards a flux.

Geometrically, tortuosity is defined as the shortest path length through a porous structure divided by its thickness. Yet, in combination with porosity, the tortuosity is also used to relate the bulk diffusion of a gas in empty space to the effective diffusion coefficient of a gas migrating through a porous membrane as shown in equation (1) (Epstein, 1989). The easiest approach to estimate tortuosity is by using porosity-tortuosity relationships (Shen and Chen, 2007), such as the widely applied Bruggeman equation (Bruggeman, 1935; Tjaden et al., 2016a), which estimate a tortuosity and thus, an effective transport property value based solely on the porosity of the structure. However, such correlations are only valid for a small number of ideal microstructures (Chung et al., 2013), which makes them invalid for the microstructures analysed here (Tjaden et al., 2016b).

$$
D_{e f f}=\frac{\varepsilon}{\tau^{2}} D_{b u l k}
$$

Due to the difficulty in determining the tortuosity of a porous membrane, a wide range of different methods have been developed in the field of electrochemistry (Tjaden et al.). Recently, improved access to tomography techniques, such as lab-based X-ray computed tomography has increased the amount of microstructural data extractable for a single sample (Izzo et al., 2008; Shearing et al., 2013b; Taiwo et al., 2016a) and has provided new ways to calculate tortuosity. However, there is a lack of standardisation across the different calculation approaches. Studies have shown that differences between imaging, simulation and experimental-based calculation methods can amount to more than a factor of two (Tjaden et al., 2016b) and might only achieve agreement under certain conditions (e.g. at high concentration losses Brus et al., 2014). One reason for this may be that image-based techniques typically do not consider all of the transport phenomena during diffusive mass transport such as Knudsen effects and are inherently limited by the imaging resolution (Finegan et al., 2016). 
Here, we present a systematic study comparing a range of tortuosity calculation algorithms of OTM porous support layers. Previously, published work by the authors (Tjaden et al., 2016b) focused on quantifying the tortuosity of OTMs through planar diffusion cell experiments at ambient temperatures. As a consequence, diffusion cell experiments are carried out at temperatures of up to $600^{\circ} \mathrm{C}$, which is close to the operating temperature of OTMs and solid oxide fuel cells (Singhal and Kendall, 2003; Delbos et al., 2010). In addition, lab-based X-ray nano computed tomography is used to capture and reconstruct the OTM microstructure in 3D for image-based calculations. The tortuosity values calculated via diffusion cell experiments of the tubular, yttria partially-stabilized zirconia (YSZ) porous support membranes are then compared with image-based simulation approaches. The effect of tortuosity, porosity and thickness of the sample structure on the membrane's resistance to diffusive mass transport is evaluated via this correlative approach and the disparity of measurements between methods is elucidated.

\section{Experimental}

\subsection{Description of Samples}

For diffusion cell experiments, four tubular YSZ porous support samples were provided by Praxair, Inc. (Tonawanda, NY, USA), which differed in porosity and wall thickness. The differences in porosities were achieved by varying the sintering temperature of the samples: Table 1 shows the different tubular samples and their respective parameters, where porosity values lay between $22.6 \%$ and $30.0 \%$ and wall thicknesses between $1.0 \mathrm{~mm}$ and $1.3 \mathrm{~mm}$. The tubular samples were cut to equal lengths of $110 \mathrm{~mm}$ for the diffusion cell experiments.

Table 1: Sample specifications of tubular porous support layers for diffusion cell experiments.

\begin{tabular}{|c|c|c|c|c|c|}
\hline $\begin{array}{c}\text { Sample } \\
\text { name }\end{array}$ & $\begin{array}{c}\text { Sintering } \\
\text { temperature } \\
{\left[{ }^{\circ} \mathrm{C}\right]}\end{array}$ & $\begin{array}{c}\text { Porosity } \\
\varepsilon \\
{[-]}\end{array}$ & $\begin{array}{c}\text { Wall thickness } \\
\delta \\
{[\mathrm{mm}]}\end{array}$ & $\begin{array}{c}\text { Inner } \\
\text { diameter } \\
{[\mathrm{mm}]}\end{array}$ & $\begin{array}{c}\text { Sample } \\
\text { length } \\
{[\mathrm{mm}]}\end{array}$ \\
\hline PS 29-1.0 & 1,400 & 0.294 & 1.0 & 0.70 & 110 \\
\hline PS 30-1.3 & 1,400 & 0.300 & 1.3 & 0.62 & 110 \\
\hline PS 25-1.0 & 1,460 & 0.251 & 1.0 & 0.66 & 110 \\
\hline PS 22-1.3 & 1,460 & 0.226 & 1.3 & 0.59 & 110 \\
\hline
\end{tabular}

The porosity values for each sample were calculated by comparing the gravimetrically determined apparent density of the sample to the density of the material. The sample names indicate the porosity and thickness of each membrane. Finally, the samples were capped off on one end to fit with the tubular diffusion cell test rig, as explained in the next section.

\subsection{Diffusion Cell Experiments}

The diffusion cell test rig layout was similar to the one used previously (Tjaden et al., 2016b), which was extended by a tubular furnace, housing the tubular diffusion cell. Figure $1 A$ and $B$ illustrate the components used and the operating principle of the diffusion cell for the tubular porous support samples, respectively. The samples were mounted via a standard 1/4" Swagelok Ultra-Torr vacuum fitting to ensure gastight sealing. A high temperature O-ring (BS012P330B, Polymax Ltd.) was inserted for experiments below $300^{\circ} \mathrm{C}$. For temperatures above $300{ }^{\circ} \mathrm{C}$, a ceramic adhesive (Ceramabond 685-N, Aremco Products, Inc.) combined with layers of Thermiculite 866 (Flexitallic 
Ltd.) was applied around the $1 / 4^{\prime \prime}$ fitting. The furnace was capable of reaching temperatures above $1,000{ }^{\circ} \mathrm{C}$, however, the highest temperature during experiments was set to $600^{\circ} \mathrm{C}$ due to the maximum operating temperature of stainless steel components of the tubular diffusion cell.

The tubular samples were sealed on one end to fit into the cell configuration shown in Figure 1: fuel gases were injected into the tubular sample by a $1 / 8^{\prime \prime}$ tube, which reached as close to the sealed end of the tubular sample as possible. The injected fuel gas was thus forced to travel back towards the $1 / 4$ " along the inside of the porous sample. The whole cell was operated inside an impervious aluminous porcelain work tube with an inner diameter of $38 \mathrm{~mm}$, mounted in a tubular furnace (EST 12/300B, Carbolite Ltd.). The void between the porous support sample and the work tube was swept with pure nitrogen, flowing counter-currently to the fuel gas on the inside of the porous membrane. The diffusion cell layout presented here is comparable to the reactor used by Delbos et al. (Delbos et al., 2010), where the performance of a tubular OTM for $\mathrm{CH}_{4}$ reforming was evaluated.
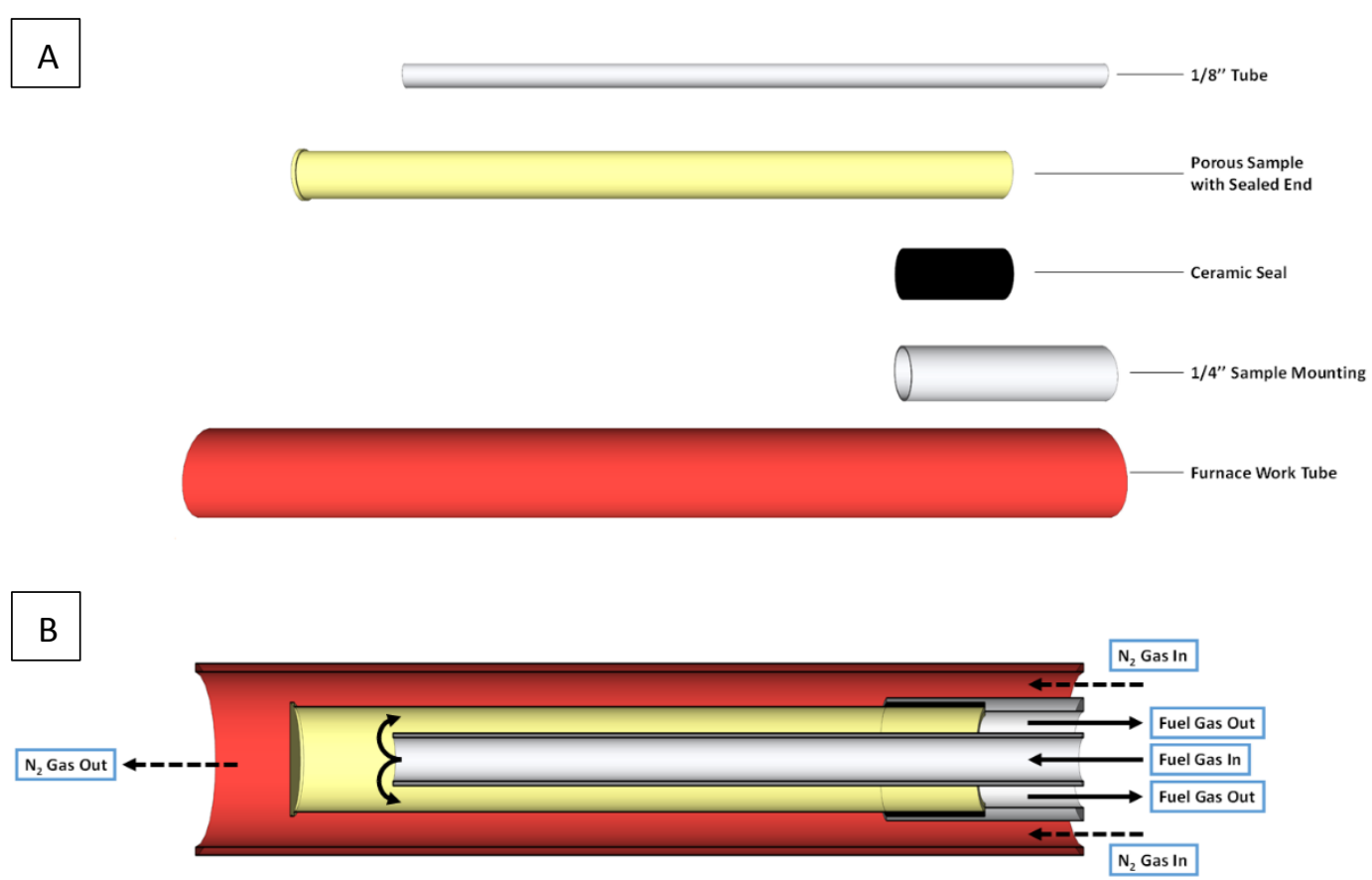

Figure 1: Components (A) and operating principle (B) of the tubular diffusion cell.

Diffusive mass transport across the porous membrane was induced by injecting pure $\mathrm{N}_{2}$ on the outside and a pure fuel gas on the inside of the porous support layer. Fuel gases considered here included $\mathrm{CH}_{4}, \mathrm{CO}$ and $\mathrm{CO}_{2}$ to be commensurate to the planned usage of the OTM for natural gas reformation and combustion (Rosen et al., 2011). Hydrogen was not tested due to the inconsistent results observed in our previous work (Tjaden et al., 2016b). The gases were supplied by a set of calibrated mass flow controllers (Bronkhorst Ltd.) at a flow rate of $100 \mathrm{~cm}^{3} \mathrm{~min}^{-1}$ on both sides of the sample.

The gas compositions in the exiting gas lines were measured using the thermal conductivity detector of a GC-2014 gas chromatograph (Shimadzu Corporation) with an argon carrier gas. The diffusion flux of each species across the sample was achieved by applying a mass balance over the diffusion cell and evaluating the gas composition of the exiting gas lines. By adjusting needle valves in the test 
rig, the pressure differences across the porous membrane was kept between $\pm 10 \mathrm{~Pa}$ to ensure no viscous flux contribution. Consequently, only ordinary and Knudsen diffusion fluxes were induced.

The dusty gas model (DGM) (eq. (2)) was employed to account for Knudsen and ordinary diffusion effects by including the Knudsen diffusion coefficient (eq. (3)) and the binary diffusion coefficient calculated via the Chapmen-Enskog correlation (Bird et al., 2002). The DGM is an implicit model (Mason and Malinauskas, 1983; Bertei and Nicolella, 2015), which can be simplified and rearranged into an explicit form valid for binary gas mixtures as presented by Liu et al. (Liu et al., 2009). The resulting equation is shown in eq. (2).

In equations (2) and (3), the effective diffusion coefficients are the respective bulk diffusion coefficients extended by the factor $\frac{\varepsilon}{\tau^{2}}$ analogue to equation (1).

$$
\begin{gathered}
J_{i, D}=-\frac{D_{i j, e f f} D_{i, K, e f f}}{D_{i j, e f f}+y_{i} D_{j, K, e f f}+y_{j} D_{i, K, e f f}} \nabla c_{i}-c_{i}\left[\frac{D_{i, K}^{e f f} D_{j, K}^{e f f}}{R T c_{t o t}\left(D_{i j, e f f}+y_{i} D_{j, K, e f f}+y_{j} D_{i, K, e f f}\right)}+\frac{B_{O}}{\mu}\right] \nabla p \\
D_{i, K n}=\frac{d_{p}}{3} \sqrt{\frac{8 R T}{\pi M_{i}}}
\end{gathered}
$$

The dusty gas model allowed the extraction of the tortuosity for each temperature by inserting the measured gas concentration and the calculated diffusion flux. It is noteworthy that two distinct diffusion fluxes were measured for each binary gas mixture as previously shown (Tjaden et al., 2016b) and thus, two tortuosity values were calculated for every binary gas mixture. Both diffusion fluxes were compared to Graham's law (Kast and Hohenthanner, 2000), shown in equation (4), to verify the consistency of the experimental setup. In these experiments, Graham's law was satisfied in all cases with an average deviation of $<10 \%$.

$$
\frac{J_{i}}{J_{j}}=\sqrt{\frac{M_{j}}{M_{i}}}
$$

\subsection{X-ray nano Computed Tomography}

X-ray nano computed tomography (nano-CT) (Stock, 2013; Maire and Withers, 2014) was used to image and reconstruct the samples in 3D. The tomograms were subsequently used as a framework for image-based tortuosity calculation approaches.

All four tubular porous support samples were imaged using the lab-based Zeiss Xradia 810 Ultra nano-CT system. In large field-of-view mode, this X-ray device achieves voxel sizes of $c a .63 .1 \mathrm{~nm}$ by the use of X-ray focussing optics (Shearing et al., 2013b), which was necessary to capture all features of the sample microstructure that affect diffusive mass transport. This high magnification is coupled with a limited field of view which amounts to $<65 \mu \mathrm{m}$.

To prepare suitable samples, $3 \mathrm{~mm}$ wide sections were cut off of each sample using a continuous rim diamond saw blade (Malvern Lapidary) on an IsoMet 11-180 low speed saw (Buehler). These sections were further reduced in size manually and sample fragments of a suitable diameter were mounted onto a needle using a two-component epoxy glue (ITW Devcon). Table 2 lists the X-ray 
nano CT imaging specifications for each of the four samples. Zernike phase contrast mode imaging was chosen over absorption mode imaging (Holzner et al., 2010; Taiwo et al., 2016b) despite the highly attenuating material, due to the better image quality in direct comparison.

Table 2: Summary of X-ray nano CT specifications for both tubular samples

\begin{tabular}{l|c|r} 
Parameter & Unit & Value \\
\hline Voxel size & {$[\mathrm{nm}]$} & 63.1 \\
\hline Camera Binning & {$[-]$} & 1 \\
\hline Field of View & {$[\mu \mathrm{m}]$} & 65 \\
\hline X-ray energy & {$[\mathrm{keV}]$} & 5.4 \\
\hline Exposure time & {$[\mathrm{s}]$} & 90 \\
\hline No. of projections & {$[-]$} & 901 \\
\hline
\end{tabular}

The collected X-ray transmission images were reconstructed into a 3D volume using commercial software (Zeiss XRM Reconstructor) that is based on a filtered back projection algorithm and segmented using a threshold segmentation approach in Avizo Fire 8 (FEI). Threshold segmentation was considered to be suitable as the samples only consisted of a single solid and pore phase and has been exploited in previous work by the authors (Tjaden et al., 2016b). Due to the high image quality achieved during X-ray nano CT, no filters were applied before or after segmentation.

For each sample (cf. Table 1), a cube with a side length of $6.31 \mu \mathrm{m}$ was extracted, which was the largest uniform cuboid size obtainable across all samples as shown in Figure 2. The continuous pore size distribution calculation approach of Münch and Holzer (Münch and Holzer, 2008) was employed to achieve the mean pore diameter for calculating the Knudsen diffusion coefficient expressed in eq. (3).

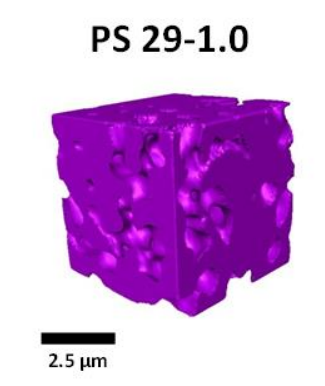

PS 25-1.0

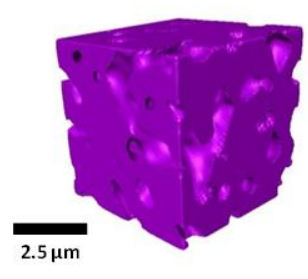

PS 30-1.3

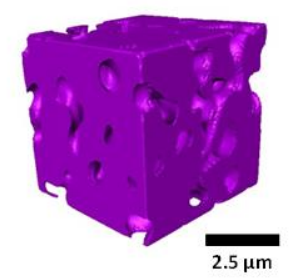

PS 22-1.3

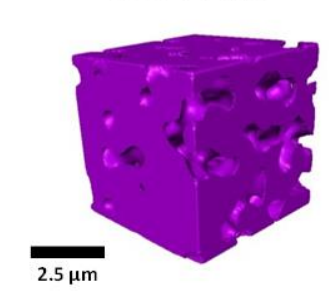

Figure 2: 3D sample cube volumes of four different porous support layer samples.

Stereological calculation approaches were applied to extract the 2D porosity and 2D pore diameter development along each axis to further analyse the distribution of microstructural parameters within the sample volume. The 3D porosity of the overall sample and the 2D porosity of each image 
slice were determined by counting the pixels of the respective phases. The $2 \mathrm{D}$ pore diameter was calculated by dividing the pore volume fraction by the interface area between the two phases (Russ and DeHoff, 2000; Exner, 2004; Finegan et al., 2016; Taiwo et al., 2016a) for each image plane along all three axes of each sample:

$$
d_{P, 2 D}=4 \frac{V_{P}}{S_{v}}
$$

\subsection{Image-based Tortuosity Calculation Approaches}

The increasing availability of tomography equipment and high performance computers has led to a significant increase in image-based microstructural analysis tools. Among these, tortuosity calculation methods have become almost standard for studying electrochemical devices (Tjaden et al.), and a wide range of methodologies has been developed.

Due to the wealth of tortuosity calculation techniques, a systematic study of tortuosity calculation procedures has been applied here for purposes of comparison. These include a flux simulation method using StarCCM+ (CD-adapco), the MATLAB (Mathworks Inc.) application TauFactor, the pore centroid method and the fast marching method (FMM). Among these, the first two approaches can be considered as flux-based algorithms while the latter two fall under the category of geometrically-based algorithms. The difference between both types is that flux-based algorithms account for constrictions and bottlenecks which affect the flux-behaviour, whereas the geometric algorithms only account for the minimal Euclidean distance. Hence, any pore connection, even a single pixel, will be included in the calculation and thus, the geometric definition of tortuosity is closely followed.

The algorithms used are briefly outlined here:

1) The flux simulation method in the StarCCM+ computational fluid dynamics package uses the analogy between Fourier's law of heat conduction and Fick's law of diffusion. The tortuosity is achieved by relating the heat flux through the porous phase of the sample to the heat flux through a dense sample volume of equal dimensions (Cooper et al., 2014; Trogadas et al., 2014; Brown et al., 2016a; Tjaden et al., 2016b).

2) The MATLAB application TauFactor uses an iterative over-relaxation method to solve the diffusion equation and, again, compares the flux through the porous phase to the flux through the dense volume of equal dimensions (Cooper et al; Brown et al., 2016b; Finegan et al., 2016).

3) The pore centroid method calculates the path length through a porous structure by following the centre of mass of pores of each 2D image slice along the in-plane direction of the volume and dividing it by the thickness of the volume (Gostovic et al., 2007; Smith et al., 2009; Shearing et al., 2012; Cooper et al., 2013; Cooper et al., 2014).

4) The fast marching method calculates the shortest path through the analysed phase by creating a distance map between the starting and end boundary of a propagating front. The shortest path between the two boundaries of the sample is then read out of the distance map and divided by the Euclidean distance of the starting and end boundary to calculate the geometric tortuosity (Jørgensen et al., 2011; Jørgensen et al., 2015; Taiwo et al., 2016a). 
It should be noted that for geometrical tortuosity calculations a value $\tau$ is calculated, whereas for flux-based methods, $\tau^{2}$ is determined, and therefore, for comparative purposes, the square root was taken where necessary. This equivalency is discussed at length by Epstein (Epstein, 1989).

The image-based tortuosity calculation approaches were carried out along each axis of the sample and thus, achieved three tortuosity values, $\tau_{x}, \tau_{y}$, and $\tau_{z}$. The characteristic tortuosity $\tau_{c}$ was calculated using equation (6) for comparative purposes (Cooper et al., 2014):

$$
\tau_{c}=3\left[\left(\tau_{x}^{-1}\right)+\left(\tau_{y}{ }^{-1}\right)+\left(\tau_{z}^{-1}\right)\right]^{-1}
$$

A representative volume element (RVE) analysis (Costanza-Robinson et al., 2011) was carried out by using a volume growing algorithm to characterise the minimum sample volume to calculate a sensible tortuosity value. This algorithm starts with a small fraction of the sample volume and sequentially increases the volume in predefined steps until reaching the overall dimensions of the sample reconstruction. For each sub-volume step, the tortuosity was calculated using both the TauFactor application and the fast marching method.

\section{Results and Discussion}

\subsection{Image-based Tortuosity}

Table 3 presents the porosity, mean pore diameter and dimensions for each extracted sample. A high degree of correlation between the image-based and gravimetrical porosities (cf. Table 1 ) is observed. Mean pore diameters follow the trend of porosity, where the high porosity samples feature a higher mean pore diameter than the low porosity samples.

Table 3: porosities, mean pore diameters and sample dimensions calculate from segmented tomograms of each sample.

\begin{tabular}{|c|c|c|c|}
\hline Sample Name & $\begin{array}{c}\text { Porosity } \varepsilon \\
{[-]}\end{array}$ & $\begin{array}{c}\text { Mean pore } \\
\text { diameter } d_{P} \\
{[\mu \mathrm{m}]}\end{array}$ & $\begin{array}{c}\text { Cube side } \\
\text { length } \\
{[\mu \mathrm{m}]}\end{array}$ \\
\hline PS 29-1.0 & 0.34 & 0.71 & \multirow{4}{*}{6.31} \\
\hline PS 30-1.3 & 0.31 & 0.73 & \\
\hline PS 25-1.0 & 0.25 & 0.66 & \\
\hline PS 22-1.3 & 0.23 & 0.63 & \\
\hline
\end{tabular}

For tortuosity calculation, both geometric methods and the TauFactor application were executed on the voxel domain. However, for the heat flux simulation, a volume mesh is required: the smoothed sample files were exported as surface mesh files from Avizo to StarCCM+, where an adaptive polyhedral volume mesh of each sample's pore phase was generated. The chosen smoothing extent in Avizo and the chosen base mesh size in StarCCM+ affect the quality of the final volume mesh and thus, the simulation results. A sensitivity analysis of both parameters was carried out; the smoothing extent was varied between 1 and 9 and the arbitrary base mesh size was increased from 0.1 to 1 . The increase in base mesh size caused a decrease of mesh cells from 600,000 to 8,000, yet, with higher smoothing extent, the necessary mesh repair steps in StarCCM+ (Cooper et al., 2014) were decreased. 


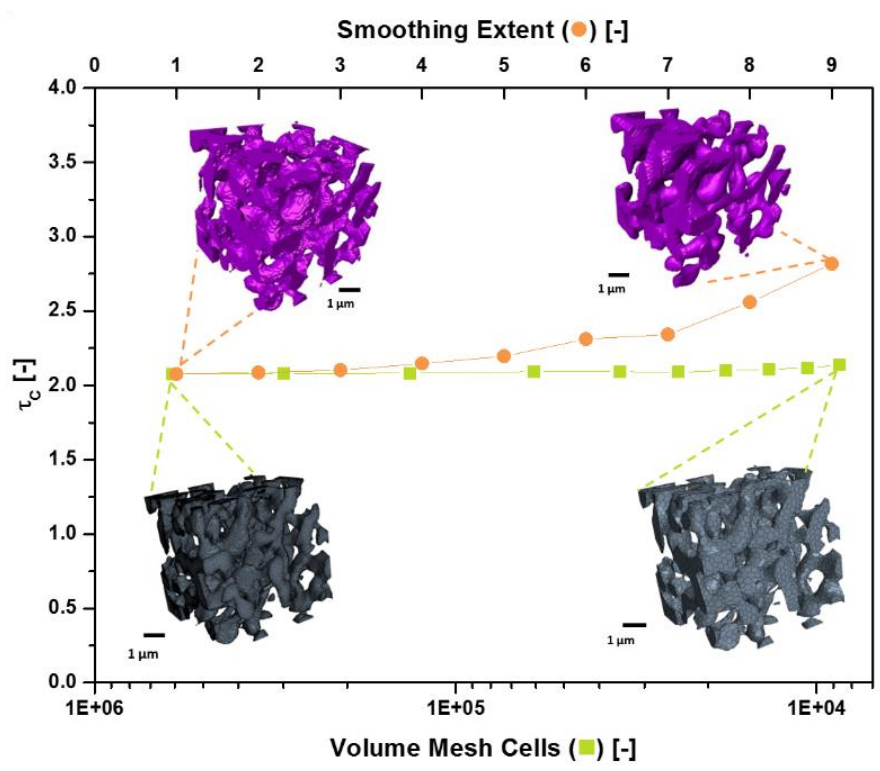

Figure 3: Effect of smoothing extent for a fixed mesh size of 0.1 and number of volume mesh elements for a fixed smoothing extent of 1 on heat flux simulation-based tortuosity of sample PS 30-1.3.

Figure 3 presents the development of characteristic tortuosity based on the heat flux simulation using the PS 30-1.3 sample cube from which it is evident that the smoothing extent has a significantly higher effect on the simulation results than the mesh size. The smoothing extent adjusts the size of the filter kernel and thus, defines how smooth the surface of the sample will be. With increasing smoothing, small features and details of the microstructure are lost which visibly affect the calculated tortuosity. By contrast, the number of volume mesh cells has only a limited effect on the calculation results. Consequently, a smoothing extent of 1 and a base mesh size of $\leq 0.2$, amounting to $>450,000$ mesh cells on all samples, were chosen for all simulations. To verify the chosen smoothing and mesh parameters, the porosity of each volume mesh was compared to the porosity calculated by pixel counting. The difference across all four sample volumes was $<3 \%$, which suggests that the chosen mesh parameters did not distort the reconstructed microstructure. 


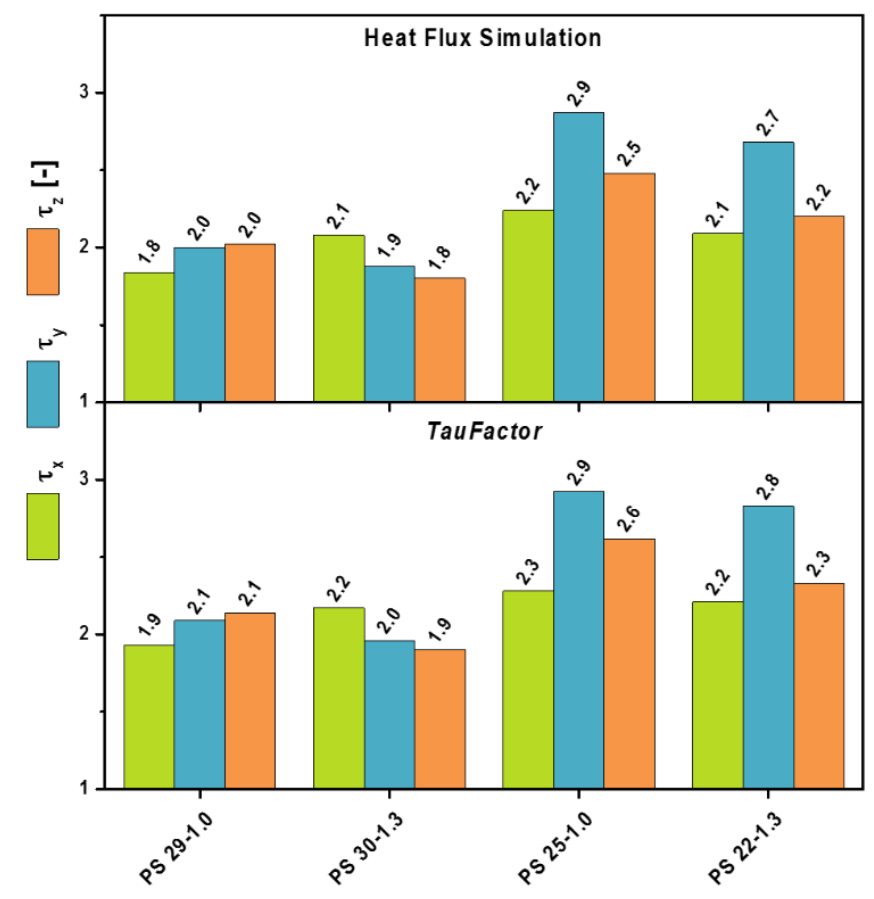

Figure 4: Flux-based tortuosity values along each axis for all four reconstructed samples.

Figure 4 compares tortuosity values along each axis for all four reconstructed samples using the heat flux simulation method and the TauFactor application. The values in Figure 4 for both calculation methods show remarkable agreement and hence, the meshing parameters of the heat flux simulation provided consistent results in comparison to the non-mesh-based algorithm. The high porosity samples (PS 29-1.0 and PS 30-1.3) feature homogeneous and low tortuosity values along each dimension. Yet, the low porosity samples (PS 25-1.0 and PS 22-1.3) exhibit higher directional anisotropy of tortuosity values for both methods. The reason for this is difficult to ascertain as all samples exhibited a pore connectivity of $>95 \%$.

To investigate the tortuosity anisotropy further, the 2D porosities of each image slice along the $x$-, $y$ - and $z$-axis for all four samples was calculated. Figure $5 \mathrm{~A}$ shows that the $2 \mathrm{D}$ porosity developments do not reveal a clear trend in either sample that could explain the directional increase in $\tau$ in samples PS 25-0 and PS 22-1.3. Instead, 2D porosities lay in a bandwidth of $\pm 10 \%$ around the average sample porosity $\left(\varepsilon_{30}\right)$. For additional exploration, the 2D pore diameter along each axis was calculated using the stereological approach of equation (5). The 2D values lie below the average 3D pore diameter results $\left(d_{P, 3 D}\right)$ as illustrated in Figure $5 \mathrm{~B}$. This is not surprising as values derived from stereological methods can differ appreciably from 3D-based values (Taiwo et al., 2016a). Moreover, the applied calculation method seems to overestimate the interface area between the two phases resulting in a lower pore diameter. As a consequence, the attention in Figure 5B is drawn to the development of the curves rather than the achieved values. It is evident that each sample's $2 \mathrm{D}$ pore diameters are comparable to each other and do not suggest elongated pores or an accumulation of constrictions in any dimension. 


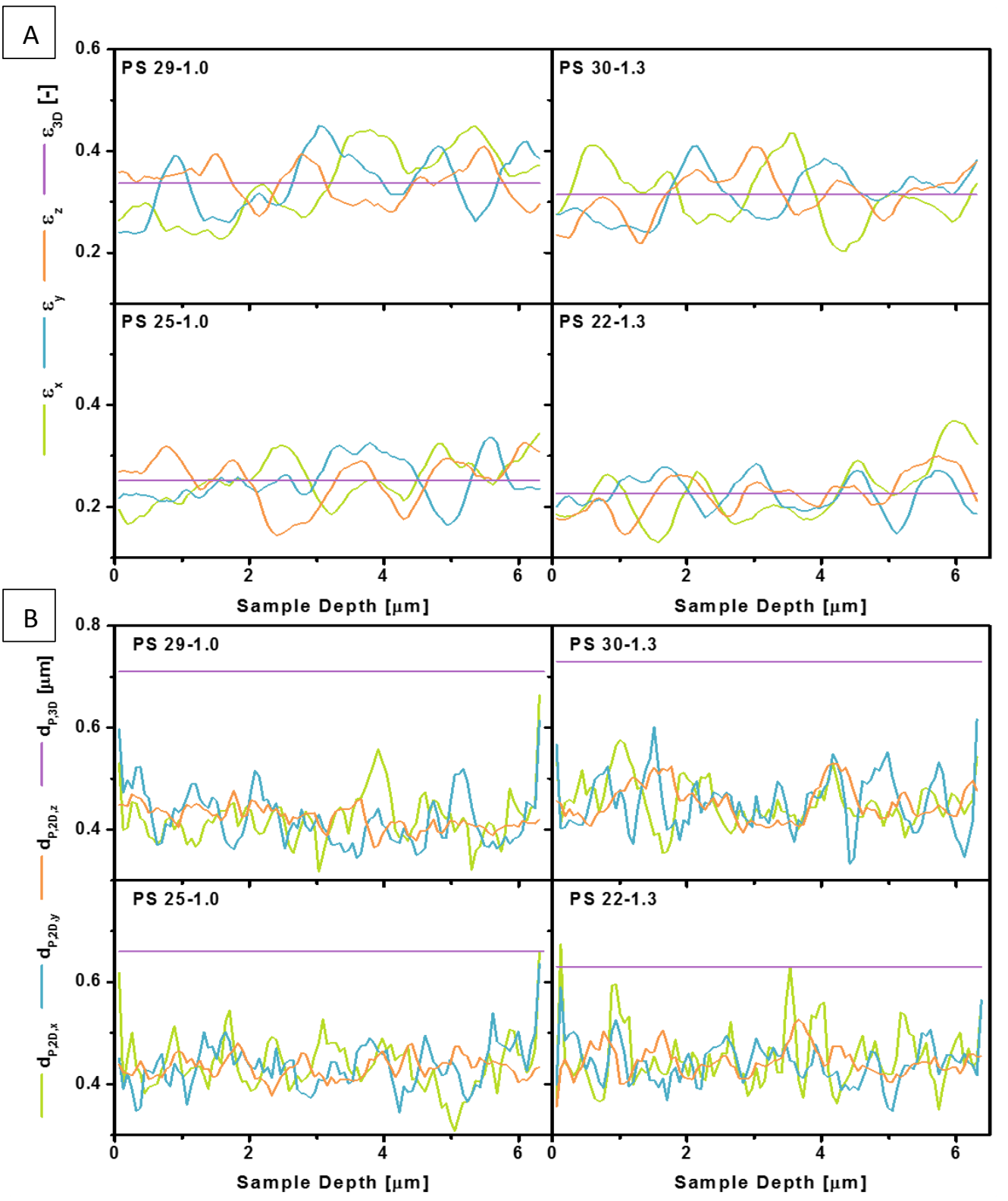

Figure 5: 2D porosity (A) and pore diameter (B) development along the $x-, y-$, and $z$-axes of each sample. 


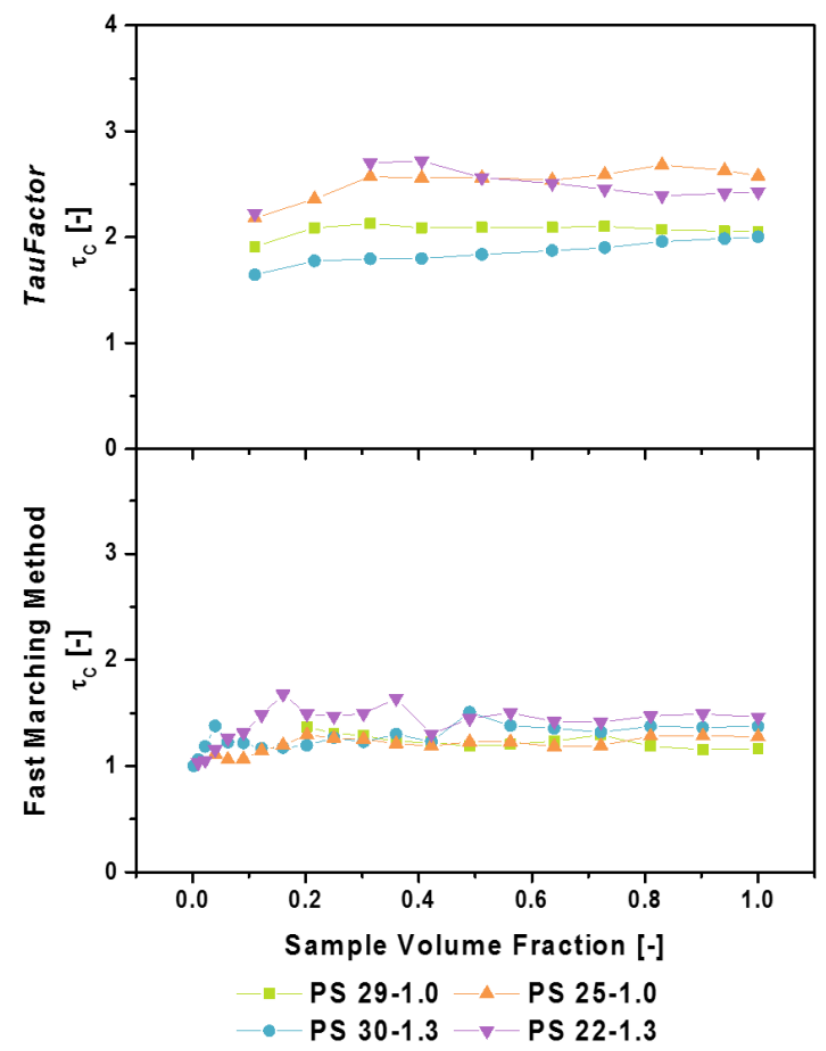

Figure 6: Representative volume element analysis of characteristic tortuosity $\tau_{c}$ for each sample using the TauFactor application and the fast marching method.

None of the microstructural analyses shown in Figure 5 explains the observed tortuosity heterogeneities encountered for samples PS 25-0 and PS 22-1.3. This highlights the complex interplay between microstructural parameters which is sometimes counter-intuitive where high porosity does not automatically guarantee a low tortuosity (Shearing et al., 2010). Indeed, anisotropies in tortuosity have been found in other porous microstructures featuring even higher porosity (Shearing et al., 2012; Cooper et al., 2014; Tariq et al., 2014).

A representative volume element analysis of tortuosity using the TauFactor application and the fast marching method was carried out to determine whether the analysed volumes were representative of the bulk. As both methods were executed in all three dimensions of the pore phase, only the characteristic tortuosity $\tau_{c}$ is shown Figure 6 . Discontinuities in the graphs signify sub-volumes, which do not achieve pore percolation across the volume, resulting in a tortuosity of infinity. All four samples achieve a flat development of $\tau_{c}$ at high sample volume fractions for both methods. The maximum deviation between the penultimate and the last value amounts to $<7 \%$. Therefore, each volume is considered to provide representative tortuosity values.

Table 4 summarises the characteristic tortuosity values of all four samples and all four image-based calculation algorithms. The flux-based approaches arrive at appreciably higher tortuosity values compared to the geometric-based fast marching method. These differences were caused by the inability of geometric methods to account for constrictions and bottlenecks, which only consider the minimal Euclidean distance between two planes. Hence, any pore connection, even a single pixel, was included in the calculation and thus, the geometric definition of tortuosity is closely followed. However, a migrating flux is affected by the variation of pore diameters within the sample and 
choses the path of least resistance and constriction. As a consequence, flux-based tortuosity calculation algorithms always resulted in a higher tortuosity value. The pore centroid method results were in between the FMM and the flux-based methods. Yet, this approach has the least physical significance in analysing the diffusion resistance of a porous structure. The algorithm locates the pore centroid of each complete image slice and follows it in the in-plane direction. However, this centroid is not necessarily located on the pore phase, but might lie on a pixel of the solid phase. Hence, this algorithm is considered only as an indicator for the homogeneity of the porous phase within the sample: the more homogeneous the sample microstructure, the closer is the centroid to the centre of the image and thus, the closer will the resulting tortuosity be to unity. The tortuosity heterogeneities of the low tortuosity samples shown in Figure 4 are thus also reflected by the higher pore centroid tortuosity values. Among the flux-based methods, the TauFactor application produced consistent results and is easiest to execute, as the binarized image sequence is sufficient as input parameter, making the generation of a volume mesh unnecessary.

It is visible, that the samples analysed here feature a constant porosity. However, this is not always the case in porous layers of electrochemical devices. For example, multi-layered battery separators can exhibit distinctive microstructural characteristics along their thickness. The image-based calculation methods presented here can be applied to evaluate the effect of such changes on the tortuosity of the overall porous layer as presented in (Finegan et al., 2016).

Table 4: Comparison of image-based characteristic tortuosity values.

\begin{tabular}{|c|c|c|c|c|}
\hline Sample Name & $\begin{array}{l}\text { Fast marching } \\
\text { method }\end{array}$ & $\begin{array}{l}\text { Pore centroid } \\
\text { method }\end{array}$ & $\begin{array}{l}\text { Heat flux } \\
\text { simulation }\end{array}$ & $\begin{array}{l}\text { TauFactor } \\
\text { application }\end{array}$ \\
\hline PS 29-1.0 & 1.27 & 1.64 & 1.95 & 2.05 \\
\hline PS 30-1.3 & 1.37 & 1.65 & 1.91 & 2.00 \\
\hline PS 25-1.0 & 1.53 & 1.75 & 2.51 & 2.58 \\
\hline PS 22-1.3 & 1.46 & 1.85 & 2.30 & 2.43 \\
\hline
\end{tabular}

\subsection{Diffusion Cell-based Tortuosity}

When consulting the image-based tortuosity calculation models and the geometric definition of tortuosity, it is suggested that tortuosity is independent of temperature. However, previous studies of diffusion through porous structures have observed conflicting results (Zamel et al., 2010; Tjaden et al., 2016b). As a consequence, the effect of temperature on the diffusion behaviour and thus, tortuosity is analysed here. Figure 7 shows the measured diffusion fluxes of each gas for temperatures between $100{ }^{\circ} \mathrm{C}$ and $600^{\circ} \mathrm{C}$. The increase in diffusion flux is almost linear for all gases and deviates only in isolated cases. The flux of $\mathrm{CO}_{2}$ for the low porosity samples drops slightly between $300{ }^{\circ} \mathrm{C}$ and $400{ }^{\circ} \mathrm{C}$ after the seal was swapped. Also, between $200^{\circ} \mathrm{C}$ and $300^{\circ} \mathrm{C}, J_{\mathrm{CH} 4}$ does not increase for both high porosity samples and at $500^{\circ} \mathrm{C}, J_{\mathrm{CH} 4}$ even decreases for sample PS 22-1.3. The reason for this behaviour is unknown but may be caused by measurement inaccuracies, which are estimated to lie between $\pm 10 \%$, analogous to the maximum deviation between the measurements and Graham's law, which was found to be $<10 \%$ in all experiments. Sealing problems are rejected as the source of error because of the consistent development of the remaining fluxes. Yet, the overall positive trend of diffusion fluxes as function of temperature is evident for all samples and binary gas mixtures. 


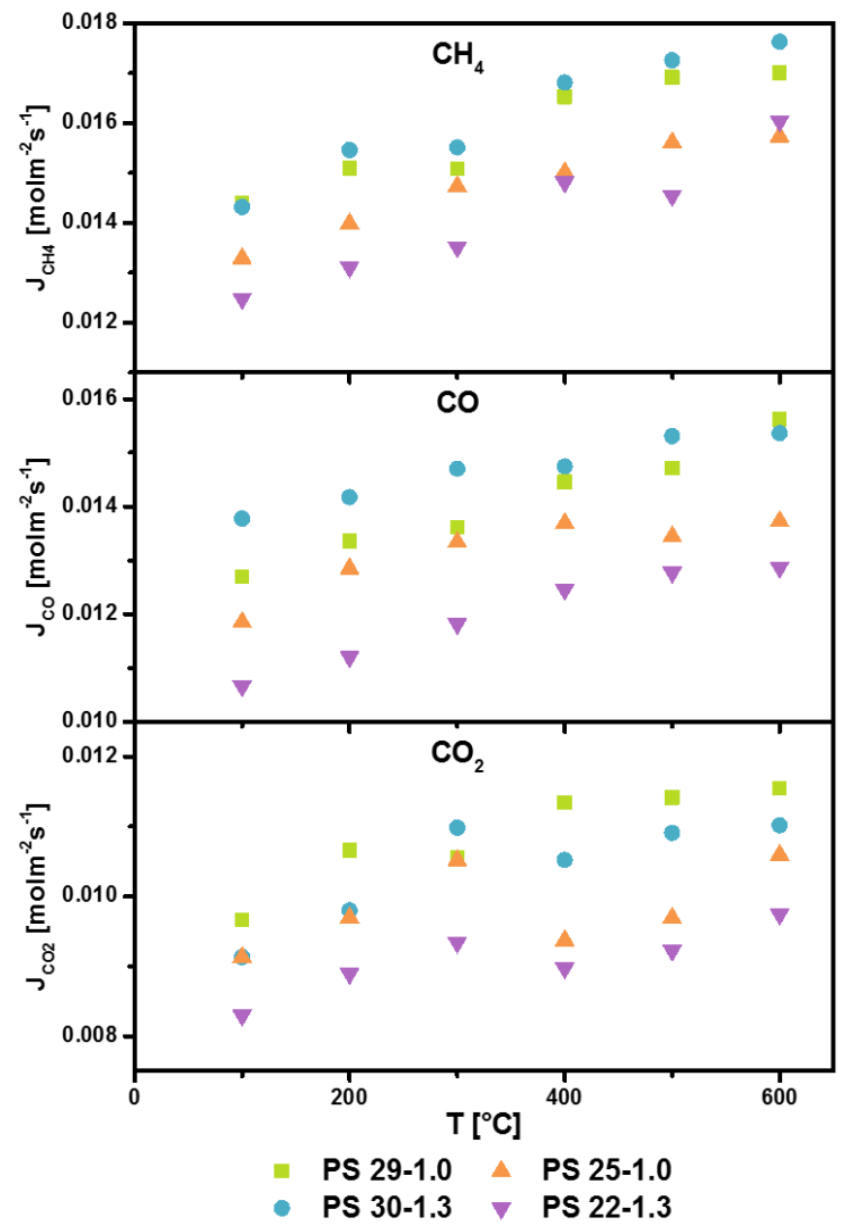

Figure 7: Diffusion flux of $\mathrm{CH}_{4}, \mathrm{CO}$ and $\mathrm{CO}_{2}$ as function of temperature for each sample.

The results illustrate that diffusion fluxes for the high porosity samples are higher than for the low porosity samples, which is in accordance with the calculated flux-based tortuosity values in Table 4 Hence, the flux-based tortuosity algorithms are capable of providing qualitative conclusions on the diffusion resistance of porous structures. Also, a clear hierarchical order of gas species is evident with $\mathrm{CO}_{2}$ achieving the lowest and $\mathrm{CH}_{4}$ the highest flux. This trend is consistent throughout the temperature range of experiments and across all samples.

Figure 8 compares the tortuosity values based on the measured diffusion fluxes for each binary gas mixture as function of temperature calculated using the dusty gas model. Despite the clear trend in increasing diffusion flux with increasing temperature, constant tortuosity values are obtained throughout the whole temperature range, which follows the conceptual definition of tortuosity closely and highlights the consistency of the diffusion cell experiments: a constant tortuosity as function of temperature suggests that the measured diffusion fluxes scale at the same rate as the calculated diffusion coefficients.

It is observed that the tortuosity values for all samples lie between 2.6 and 3.3. The average diffusion cell-based tortuosity values across all temperatures and binary gases for each sample lie very close to each other, as shown in Table 5: hardly any difference in the average tortuosity between the different samples is recognized despite the appreciable variation in porosity and measured diffusion flux. This might be explained by the small mean pore diameters of the samples: porosity and mean pore diameter between samples PS 22-1.3 and PS 30-1 increase by a factor of 1.33 and 1.15, 
respectively, while the diffusion flux of $\mathrm{CH}_{4}$, on average, only increases by a factor of 1.13 . Hence, it is apparent that the diffusion flux is governed predominantly by the pore diameter, which is comparable across all samples (cf. Table 3), rather than the porosity alone. As a consequence, Knudsen diffusion effects might be considerable within these samples. Even though the dusty gas model includes the Knudsen diffusion coefficients, the effect of varying pore diameter within the sample might not be fully captures by the mean pore diameter used for calculations. This might also explain the appreciable difference between the diffusion cell experiment tortuosity values and the image-based results shown in the next section. The effect of varying pore diameter on the diffusion behaviour has been recently analysed and published (Lu et al., 2017).

It has to be pointed out that during OTM operation reforming reactions are continuously taking place on the anode side of the membrane. Such reactions maintain a high concentration gradient (Delbos et al., 2010) in contrast to the diffusion cell experiments presented here, which might affect the diffusion behaviour and thus, the calculated tortuosity of the sample. Hence, for quantitative analyses, the diffusion cell experimental approach provides a tortuosity value dominating under the current experimental conditions. These conditions, however, might have a substantial influence on the calculated tortuosity, as shown in (Brus et al., 2014): SOFC anode tortuosity values as low as 2 and above 12 were achieved depending on the injected fuel gas concentration and degree of concentration polarisation. As a consequence, following previous observations in literature (Tsai and Schmidt, 2011; Brus et al., 2014), it is expected that the tortuosity values presented in Table 5 will decrease to values similar to the ones calculated using flux-based approaches when the complete oxygen transport membrane is in operation under high fuel conversation ratios, where mass transport limitations become more dominating.

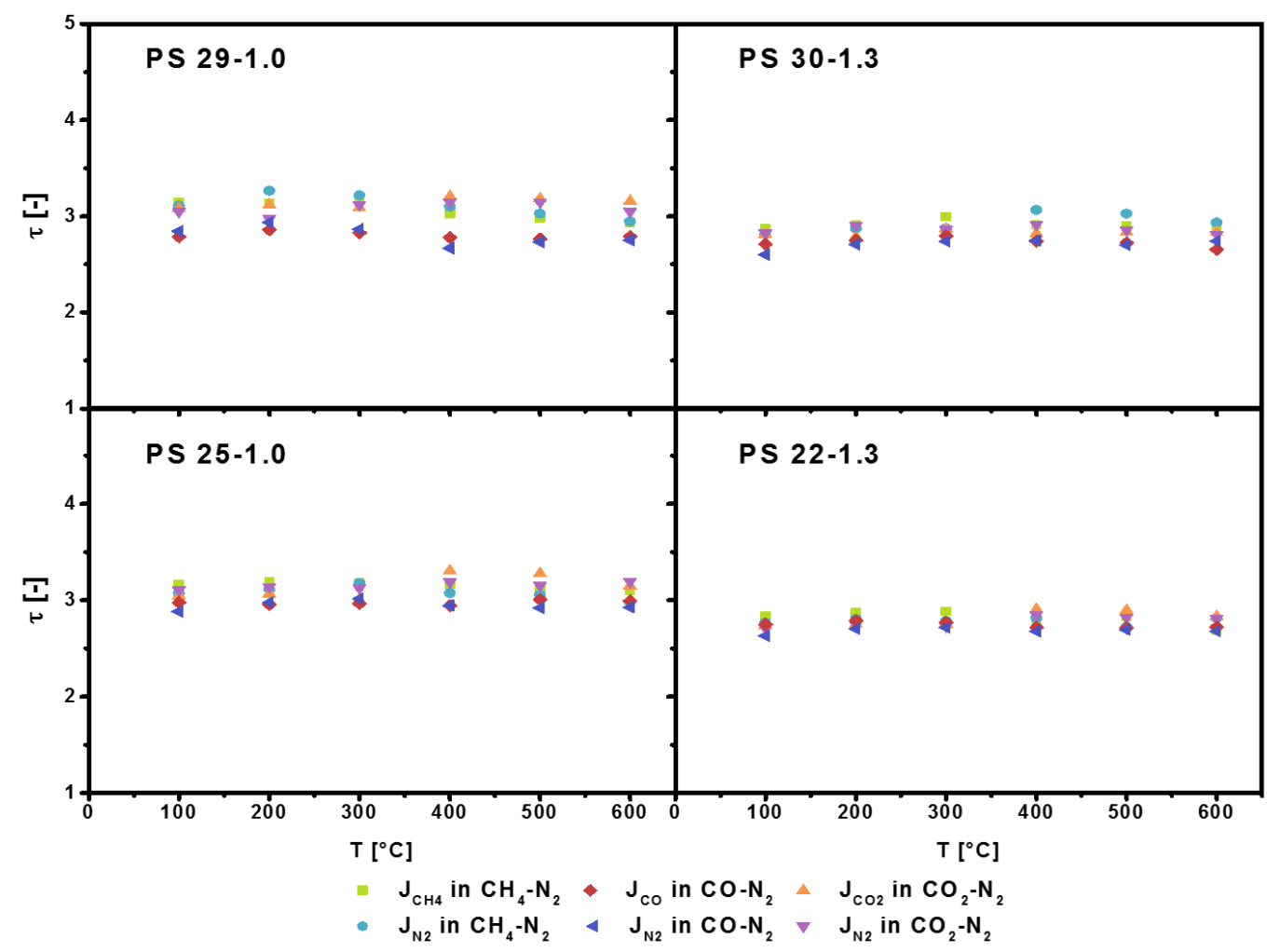

Figure 8: Tortuosity values for each sample for three different binary gas mixtures across a range of temperatures. 
Finally, the tortuosity values of samples PS $2.430 .0 \%$ and PS 29-1.0 were compared to evaluate the effect of sample thickness on the tortuosity: both of these samples feature an almost identical porosity and mean pore diameter, making the thickness the distinguishing parameter. Table 5 revealed only a minor effect of the thickness of the samples on the tortuosity which is consistent with the definition of tortuosity of being a microstructural characteristic independent of operating condition and scale and analogue to observations presented in (Tsai and Schmidt, 2011). This is perhaps not surprising, as the experimental measurements were carried out at steady-state, but might be more significant in transient diffusion regimes.

Table 5: Comparison of porous support layer properties calculated using average tortuosity values from diffusion cell experiments.

\begin{tabular}{|c|ccc}
$\begin{array}{c}\text { Sample } \\
\text { Name }\end{array}$ & $\begin{array}{c}\text { Porosity } \boldsymbol{\varepsilon} \\
\text { taken from } \\
\text { Table } 1 \\
{[-]}\end{array}$ & $\begin{array}{c}\text { Wall } \\
\text { thickness } \boldsymbol{\delta} \\
{[\boldsymbol{\mu} \boldsymbol{m}]}\end{array}$ & $\begin{array}{c}\text { Average } \\
\text { diffusion } \\
\text { cell-based } \\
\text { tortuosity } \boldsymbol{\tau} \\
{[-]}\end{array}$ \\
\hline PS 29-1.0 & 0.294 & 1,000 & 3.0 \\
\hline PS 30-1.3 & 0.300 & 1,300 & 2.8 \\
\hline PS 25-1.0 & 0.251 & 1,000 & 3.1 \\
\hline PS 22-1.3 & 0.226 & 1,300 & 2.8 \\
\hline
\end{tabular}

\subsection{Comparison of Results}

Figure 9 compares the characteristic tortuosity values of the image-based calculation algorithms with average tortuosity values across all experiments from Figure 8 for all four porous support samples. The error bars in Figure 9 indicate the range between the minimum and maximum tortuosity values achieved for each sample. A clear hierarchy in results is observed: geometric calculation approaches including the FMM and the pore centroid method provide the lowest, while the diffusion cell experiments provide the highest tortuosity values. Results of the flux-based models such as the heat flux simulation and the TauFactor application lie in between. Among the image-based algorithms, the flux-based approaches are more physically sound due to the consideration of constrictions and bottlenecks during calculations. Thus, the conceptual migration of a flux is closely followed. Here, the TauFactor application presents itself as the easiest method to extract a meaningful tortuosity value due to its simplicity to use. Moreover, flux-based tortuosity calculation methods are easy to reproduce across different samples, independent of sample type, architecture and functionality and thus, serve as an excellent base for qualitative comparisons of microstructural parameters.

While the flux-based values feature a clear variation in tortuosity according to each sample's porosity, such an effect is not noticeable, for geometric tortuosity nor for the diffusion cell values. As mentioned previously, Knudsen effects might be appreciable due to the small pore diameters of the treated sample structures. Whilst Knudsen diffusion is included in the diffusion cell experiments, the local variation in pore diameter might be substantial. Moreover, Knudsen effects are not implemented in the image-based tortuosity calculation methods at all, which might be the reason for the diverging tortuosity values. 


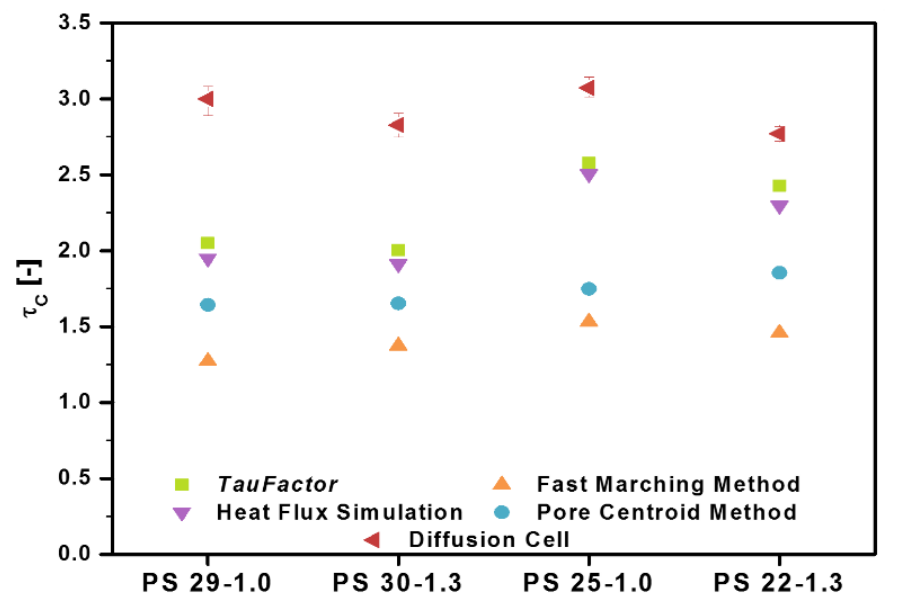

Figure 9: Comparison of geometric, simulation and diffusion cell-based tortuosity values for all four porous support samples.

The conclusions drawn from image and experimental methods coincide when considering the measured diffusion fluxes shown in Figure 7: the porous support layer should feature both high porosity and high thickness to ensure both high mechanical stability and high mass transport performance. As a consequence, it is recommended that for steady-state operation the sample thickness of the porous support layer should be increased rather than decreasing its porosity to improve the mechanical membrane stability. However, the effect of sample thickness might become more apparent in transient operation though for example during start-up and shut-down of the OTM reactor, which has not yet been evaluated for the samples at hand.

\section{Conclusions}

Here, we analysed the mass transport resistance of four yttria partially-stabilized zirconia porous support samples for application in oxygen transport membranes by calculating the tortuosity using image and diffusion cell-based approaches. A clear hierarchy in calculated values was revealed, where geometric methods achieved lowest, and diffusion cell experiments achieved highest tortuosities. Flux-based simulation models were in between. The image-based analyses showed that high porosity samples provide lowest tortuosity values which is in agreement with the measured diffusion fluxes. However, using diffusion cell experiments, no clear dependency between tortuosity and porosity was obvious over the whole range of analysed temperatures and gas mixtures. The discrepancy between image and diffusion cell-based values might stem from the small pore diameter of the samples, which seemed to have a higher impact on the measured diffusion flux than the porosity alone, highlighting the importance of Knudsen diffusion effects in the analysed samples. Yet, flux-based models serve as an easily applied tool to qualitatively compare the tortuosity of a porous structures.

For future work, the effect of the thickness of the porous support layer on the diffusion performance in transient operation, for example during start-up or shutdown procedures of the reactor, will be evaluated. For this, mass spectrometry, which allows the measurement of gas concentration in real-time, will be employed to obtain a complete picture of mass transport phenomena at steady-state and during transient operation. 


\section{Nomenclature}

$\begin{array}{ll}\varepsilon & \text { Porosoty (-) } \\ \mu & \text { Dynamic viscosity }\left(\mathrm{kgm}^{-1} \mathrm{~s}^{-1}\right) \\ \tau & \text { Tortuosity (-) } \\ & \\ B_{O} & \text { Viscous flow parameter }\left(\mathrm{m}^{2}\right) \\ c_{i} & \text { Molar concentration }\left(\mathrm{molm} \mathrm{m}^{-3}\right) \\ D_{\text {bulk }} & \text { Bulk diffusion coefficient }\left(\mathrm{m}^{2} \mathrm{~s}^{-1}\right) \\ D_{e f f} & \text { Effective diffusion coefficien }\left(\mathrm{m}^{2} \mathrm{~s}^{-1}\right) \\ D_{i, K, e f f} \text { and } D_{j, K, e f f} & \text { Effective Knudsen diffusion coefficients }\left(\mathrm{m}^{2} \mathrm{~s}^{-1}\right) \\ D_{i, K n} & \text { Knudsen diffusion coefficient }\left(\mathrm{m}^{2} \mathrm{~s}^{-1}\right) \\ D_{i j, e f f} & \text { Effective binary diffusion coefficient }\left(\mathrm{m}^{2} \mathrm{~s}^{-1}\right) \\ d_{P} & \text { Mean pore diameter }(\mathrm{m}) \\ d_{P, 2 D} & \text { 2D pore diameter } \\ J_{i, D} & \left.\text { Diffusion flux (molm }{ }^{-2} \mathrm{~s}^{-1}\right) \\ M_{i} & \left.\text { Molar mass (kgmol }{ }^{-1}\right) \\ p & \text { Pressure (Pa) } \\ R & \text { Ideal gas constant }\left(\mathrm{Jmol}^{-1} \mathrm{~K}^{-1}\right) \\ S_{V} & \text { 2D interface area between the two phases } \\ T & \text { Temperature (K) } \\ V_{P} & \text { 2D pore volume fraction } \\ y_{i} \text { and } y_{j} & \text { Molar fractions }(-)\end{array}$

\section{Acknowledgments}

The authors acknowledge Praxair Inc. for financial support of this work and the EPSRC (EP/M014045/1, EP/L015749/1). Equipment access was supported by UCL and the EPSRC (EP/K005030/1). PRS acknowledges the Royal Academy of Engineering for their support.

\section{References}

Bertei, A., Nicolella, C., 2015. Common inconsistencies in modeling gas transport in porous electrodes: The dusty-gas model and the Fick law. J. Power Sources 279, 133-137. 10.1016/j.jpowsour.2015.01.007.

Bertei, A., Nucci, B., Nicolella, C., 2013. Microstructural modeling for prediction of transport properties and electrochemical performance in SOFC composite electrodes. Chem. Eng. Sci. 101 (0), 175-190. 10.1016/j.ces.2013.06.032.

Bird, R.B., Stewart, W.E., Lightfoot, E.N., 2002. Transport phenomena, 2nd ed. J. Wiley, New York. Brown, L.D., Abdulaziz, R., Tjaden, B., Inman, D., Brett, D.J.L., Shearing, P.R., 2016a. Investigating microstructural evolution during the electroreduction of $\mathrm{UO} 2$ to $\mathrm{U}$ in $\mathrm{LiCl}-\mathrm{KCl}$ eutectic using focused ion beam tomography. Journal of Nuclear Materials 480, 355-361. 10.1016/j.jnucmat.2016.07.036. 
Brown, L.D., Neville, T.P., Jervis, R., Mason, T.J., Shearing, P.R., Brett, D.J.L., 2016b. The effect of felt compression on the performance and pressure drop of all-vanadium redox flow batteries. Journal of Energy Storage 8, 91-98. 10.1016/j.est.2016.10.003.

Bruggeman, D.A.G., 1935. Berechnung verschiedener physikalischer Konstanten von heterogenen Substanzen. I. Dielektrizitätskonstanten und Leitfähigkeiten der Mischkörper aus isotropen Substanzen. Ann. Phys. 416 (7), 636-664. 10.1002/andp.19354160705.

Brus, G., Miyawaki, K., Iwai, H., Saito, M., Yoshida, H., 2014. Tortuosity of an SOFC anode estimated from saturation currents and a mass transport model in comparison with a real micro-structure. Solid State Ionics 265, 13-21. 10.1016/j.ssi.2014.07.002.

Chen, Z., Wang, X., Giuliani, F., Atkinson, A., 2015. Microstructural characteristics and elastic modulus of porous solids. Acta Mater. 89, 268-277. 10.1016/j.actamat.2015.02.014.

Chung, D.-W., Ebner, M., Ely, D.R., Wood, V., García, E.R., 2013. Validity of the Bruggeman relation for porous electrodes. Modell. Simul. Mater. Sci. Eng. 21 (7), 74009.

Cooper, S.J., Bertei, A., Shearing, P.R., Kilner, J.A., Brandon, N.P. TauFactor: An open-source application for calculating tortuosity factors from tomographic data. SoftwareX. 10.1016/j.softx.2016.09.002.

Cooper, S.J., Eastwood, D.S., Gelb, J., Damblanc, G., Brett, D.J.L., Bradley, R.S., Withers, P.J., Lee, P.D., Marquis, A.J., Brandon, N.P., Shearing, P.R., 2014. Image based modelling of microstructural heterogeneity in LiFePO4 electrodes for Li-ion batteries. J. Power Sources 247, 1033-1039. 10.1016/j.jpowsour.2013.04.156.

Cooper, S.J., Kishimoto, M., Tariq, F., Bradley, R.S., Marquis, A.J., Brandon, N.P., Kilner, J.A., Shearing, P.R., 2013. Microstructural Analysis of an LSCF Cathode Using In Situ Tomography and Simulation. ECS Trans. 57 (1), 2671-2678. 10.1149/05701.2671ecst.

Costanza-Robinson, M.S., Estabrook, B.D., Fouhey, D.F., 2011. Representative elementary volume estimation for porosity, moisture saturation, and air-water interfacial areas in unsaturated porous media: Data quality implications. Water Resour. Res. 47 (7), W07513. 10.1029/2010WR009655.

Delbos, C., Lebain, G., Richet, N., Bertail, C., 2010. Performances of tubular La0.8Sr0.2Fe0.7Ga0.303- $\delta$ mixed conducting membrane reactor for under pressure methane conversion to syngas. Catal. Today 156 (3-4), 146-152. 10.1016/j.cattod.2010.04.047.

Epstein, N., 1989. On tortuosity and the tortuosity factor in flow and diffusion through porous media. Chem. Eng. Sci. 44 (3), 777-779. 10.1016/0009-2509(89)85053-5.

Exner, H.E., 2004. Stereology And 3d Microscopy: Useful Alternatives Or Competitors In The Quantitative Analysis Of Microstructures? Image Anal Stereol 23 (2), 73-82. 10.5566/ias.v23.p73-82.

Finegan, D.P., Cooper, S.J., Tjaden, B., Taiwo, O.O., Gelb, J., Hinds, G., Brett, D.J.L., Shearing, P.R., 2016. Characterising the structural properties of polymer separators for lithium-ion batteries in 3D using phase contrast X-ray microscopy. J. Power Sources 333, 184-192. 10.1016/j.jpowsour.2016.09.132.

Gostovic, D., Smith, J.R., Kundinger, D., Jones, K.S., Wachsman, E.D., 2007. Three-Dimensional Reconstruction of Porous LSCF Cathodes. Electrochem. Solid-State Lett. 10 (12), B214. 10.1149/1.2794672.

Holzner, C., Feser, M., Vogt, S., Hornberger, B., Baines, S.B., Jacobsen, C., 2010. Zernike phase contrast in scanning microscopy with X-rays. Nat Phys 6 (11), 883-887. 10.1038/nphys1765.

Izzo, J.R., Joshi, A., Grew, K., Chiu, W.K.S., Tkachuk, A., Wang, S., Yun, W., 2008. Non-destructive Reconstruction and Analysis of Solid Oxide Fuel Cell Anodes using X-ray Computed Tomography at sub-50 nm Resolution. ECS Trans. 13 (6), 1-11. 10.1149/1.2992597.

Jørgensen, P.S., Ebbeh øj, S.L., Hauch, A., 2015. Triple phase boundary specific pathway analysis for quantitative characterization of solid oxide cell electrode microstructure. J. Power Sources 279, 686-693. 10.1016/j.jpowsour.2015.01.054. 
Jørgensen, P.S., Hansen, K.V., Larsen, R., Bowen, J.R., 2011. Geometrical characterization of interconnected phase networks in three dimensions. J. Microsc. 244 (1), 45-58. 10.1111/j.13652818.2011.03504.x.

Kaiser, A., Foghmoes, S.P., Pećanac, G., Malzbender, J., Chatzichristodoulou, C., Glasscock, J.A., Ramachandran, D., Ni, D.W., Esposito, V., Søgaard, M., Hendriksen, P.V., 2016. Design and optimization of porous ceramic supports for asymmetric ceria-based oxygen transport membranes. Journal of Membrane Science 513, 85-94. 10.1016/j.memsci.2016.04.016.

Kast, W., Hohenthanner, C.-R., 2000. Mass transfer within the gas-phase of porous media. Int. J. Heat Mass Transfer 43 (5), 807-823. 10.1016/S0017-9310(99)00158-1.

Liu, S., Kong, W., Lin, Z., 2009. Three-dimensional modeling of planar solid oxide fuel cells and the rib design optimization. J. Power Sources 194 (2), 854-863. 10.1016/j.jpowsour.2009.06.056.

Lu, X., Tjaden, B., Bertei, A., Li, T., Li, K., Brett, D., Shearing, P., 2017. 3D Characterization of Diffusivities and its Impact Mass Flux and Concentration Overpotential in SOFC Anode. J. Electrochem. Soc. 164 (4), F188-F195. 10.1149/2.0111704jes.

Maire, E., Withers, P.J., 2014. Quantitative X-ray tomography. Int. Mater. Rev. 59 (1), 1-43. 10.1179/1743280413Y.0000000023.

Mason, E., Malinauskas, A., 1983. Gas transport in porous media: The dusty-gas model. Elsevier, Amsterdam, New York.

Münch, B., Holzer, L., 2008. Contradicting Geometrical Concepts in Pore Size Analysis Attained with Electron Microscopy and Mercury Intrusion. Journal of the American Ceramic Society 91 (12), 4059-4067. 10.1111/j.1551-2916.2008.02736.x.

Robertson, I., Holzer, L., Prestat, M., Münch, B., Graule, T., 2010. Effects of particle and pore sizes, surface area and porosity on the performance of LSC cathodes. Proceedings of the 9th European Fuel Cell Forum, 10-83.

Rosen, L., Degenstein, N., Shah, M., Wilson, J., Kelly, S., Peck, J., Christie, M., 2011. Development of oxygen transport membranes for coal-based power generation. 10th International Conference on Greenhouse Gas Control Technologies 4 (0), 750-755. 10.1016/j.egypro.2011.01.115.

Russ, J., DeHoff, R.T., 2000. Practical Stereology, 2nd ed. Kluwer Academic/Plenum, New York, London.

Shearing, P.R., Brandon, N.P., Gelb, J., Bradley, R.S., Withers, P.J., Marquis, A.J., Cooper, S.J., Harris, S.J., 2012. Multi Length Scale Microstructural Investigations of a Commercially Available Li-Ion Battery Electrode. J. Electrochem. Soc. 159 (7), A1023. 10.1149/2.053207jes.

Shearing, P.R., Brett, D.J.L., Brandon, N.P., 2013a. Towards intelligent engineering of SOFC electrodes: a review of advanced microstructural characterisation techniques. Int. Mater. Rev. 55 (6), 347-363. 10.1179/095066010X12777205875679.

Shearing, P.R., Eastwood, D.S., Bradley, R.S., Gelb, J., Cooper, S.J., Tariq, F., Brett, D.J.L., Brandon, N.P., Withers, P.J., Lee, P.D., 2013b. Exploring electrochemical devices using X-ray microscopy: 3D microstructure of batteries and fuel cells. Microscopy and Analysis 27 (2), 19-22.

Shearing, P.R., Howard, L.E., Jørgensen, P.S., Brandon, N.P., Harris, S.J., 2010. Characterization of the 3-dimensional microstructure of a graphite negative electrode from a Li-ion battery. Electrochem. Commun. 12 (3), 374-377. 10.1016/j.elecom.2009.12.038.

Shen, L., Chen, Z., 2007. Critical review of the impact of tortuosity on diffusion. Chem. Eng. Sci. 62 (14), 3748-3755. 10.1016/j.ces.2007.03.041.

Singhal, S.C., Kendall, K., 2003. High-temperature solid oxide fuel cells: Fundamentals, design, and applicatons. Elsevier Advanced Technology, New York.

Smith, J.R., Chen, A., Gostovic, D., Hickey, D., Kundinger, D., Duncan, K.L., DeHoff, R.T., Jones, K.S., Wachsman, E.D., 2009. Evaluation of the relationship between cathode microstructure and electrochemical behavior for SOFCs. Solid State lonics 180 (1), 90-98. 10.1016/j.ssi.2008.10.017.

Stock, S.R., 2013. Recent advances in X-ray microtomography applied to materials. Int. Mater. Rev. 53 (3), 129-181. 10.1179/174328008X277803. 
Taiwo, O.O., Finegan, D.P., Eastwood, D.S., Fife, J.L., Brown, L.D., Darr, J.A., Lee, P.D., Brett, D.J.L., Shearing, P.R., 2016a. Comparison of three-dimensional analysis and stereological techniques for quantifying lithium-ion battery electrode microstructures. J. Microsc. 263 (3), 280-292. 10.1111/jmi.12389.

Taiwo, O.O., Finegan, D.P., Gelb, J., Holzner, C., Brett, D.J.L., Shearing, P.R., 2016b. The use of contrast enhancement techniques in X-ray imaging of lithium-ion battery electrodes. Chem. Eng. Sci. 154, 27-33. 10.1016/j.ces.2016.04.023.

Tariq, F., Yufit, V., Kishimoto, M., Shearing, P.R., Menkin, S., Golodnitsky, D., Gelb, J., Peled, E., Brandon, N.P., 2014. Three-dimensional high resolution X-ray imaging and quantification of lithium ion battery mesocarbon microbead anodes. J. Power Sources 248, 1014-1020. 10.1016/j.jpowsour.2013.08.147.

Tjaden, B., Cooper, S.J., Brett, D.J.L., Kramer, D., Shearing, P.R., 2016a. On the origin and application of the Bruggeman correlation for analysing transport phenomena in electrochemical systems. Curr. Opin. Chem. Eng. 12, 44-51. 10.1016/j.coche.2016.02.006.

Tjaden, B., Lane, J., Withers, P.J., Bradley, R.S., Brett, D.J.L., Shearing, P.R., 2016b. The application of 3D imaging techniques, simulation and diffusion experiments to explore transport properties in porous oxygen transport membrane support materials. Solid State lonics 288, 315-321. 10.1016/j.ssi.2016.01.030.

Tjaden, B., Shearing, P.R., Brett, D.J.L. Understanding tortuosity in electrochemical devices: A review of calculation approaches. Int. Mater. Rev. Accepted. 10.1080/09506608.2016.1249995.

Trogadas, P., Taiwo, O.O., Tjaden, B., Neville, T.P., Yun, S., Parrondo, J., Ramani, V., Coppens, M.-C., Brett, D.J.L., Shearing, P.R., 2014. X-ray micro-tomography as a diagnostic tool for the electrode degradation in vanadium redox flow batteries. Electrochem. Commun. 48 (0), 155-159. 10.1016/j.elecom.2014.09.010.

Tsai, C.-L., Schmidt, H.V., 2011. Tortuosity in anode-supported proton conductive solid oxide fuel cell found from current flow rates and dusty-gas model. J. Power Sources 196 (2), 692-699. 10.1016/j.jpowsour.2010.08.005.

Virkar, A.V., Chen, J., Tanner, C.W., Kim, J.-W., 2000. The role of electrode microstructure on activation and concentration polarizations in solid oxide fuel cells. Solid State lonics 131 (1-2), 189-198. 10.1016/S0167-2738(00)00633-0.

Wilson, J.R., Barnett, S.A., 2008. Solid Oxide Fuel Cell Ni-YSZ Anodes: Effect of Composition on Microstructure and Performance. Electrochem. Solid-State Lett. 11 (10), B181. 10.1149/1.2960528.

Yuan, J., Sundén, B., 2014. On mechanisms and models of multi-component gas diffusion in porous structures of fuel cell electrodes. Int. J. Heat Mass Transfer 69 (0), 358-374. 10.1016/j.ijheatmasstransfer.2013.10.032.

Zamel, N., Astrath, N., Li, X., Shen, J., Zhou, J., Astrath, F., Wang, H., Liu, Z.-S., 2010. Experimental measurements of effective diffusion coefficient of oxygen-nitrogen mixture in PEM fuel cell diffusion media. Chem. Eng. Sci. 65 (2), 931-937. 10.1016/j.ces.2009.09.044. 